\title{
On some permutable products of supersoluble groups
}

\author{
Manuel J. Alejandre, A. Ballester-Bolinches, \\ John Cossey and M. C. Pedraza-Aguilera
}

\begin{abstract}
It is well known that a group $G=A B$ which is the product of two supersoluble subgroups $A$ and $B$ is not supersoluble in general. Under suitable permutability conditions on $A$ and $B$, we show that for any minimal normal subgroup $N$ both $A N$ and $B N$ are supersoluble. We then exploit this to establish some sufficient conditions for $G$ to be supersoluble.
\end{abstract}

\section{Introduction}

All groups considered in this paper will be finite. It is well known that, even for a saturated formation $\mathcal{F}$, a group that is the product of two subgroups in $\mathcal{F}$ need not to be in $\mathcal{F}$. The structure of such products and conditions which ensure that the product is in $\mathcal{F}$ have been widely studied for the classes $\mathcal{N}$ of nilpotent groups and $\mathcal{U}$ of supersoluble groups.

The behaviour of minimal normal subgroups of factorized groups has been an important source of information about their structure. Our interest is in extending the following theorem of Stonehewer [12]:

Let $G$ be a finite group which can be written as the product $G=A B$ of two nilpotent subgroups $A$ and $B$. If $N$ is a minimal normal subgroup of $G$, then either $A N$ or $B N$ is nilpotent.

Unfortunately, this result is not true if we replace nilpotent by supersoluble in the statement $(P S L(2,7)$ can be written as the product of two supersoluble subgroups). One of the main purposes of this paper is to 
find conditions which allow us to establish a supersoluble version of Stonehewer's Theorem. We then exploit these results to obtain the supersolubility of some products of supersoluble groups.

Much recent work on products of groups and supersolubility has focussed on products when the factors satisfy extra permutability conditions. The first step in this direction was taken by Baer [4].

Let $G=A B$ be the product of the supersoluble normal subgroups $A$ and $B$ of $G$. If the derived subgroup $G^{\prime}$ of $G$ is nilpotent, then $G$ is supersoluble.

Many generalizations of this theorem have been obtained. The aim of most of them has been to weaken the normality hypotheses, replacing them by permutability conditions instead. Following Carocca [7], we will say that:

$A$ group $G$ is said to be the totally permutable product (t.p.p.) of the subgroups $A$ and $B$ if $G=A B$ and every subgroup of $A$ permutes with every subgroup of $B$.

The product $G=A B$ is said to be mutually permutable (m.p.p.) if $A$ permutes with every subgroup of $B$ and $B$ permutes with every subgroup of $A$.

Every totally permutable product is clearly mutually permutable, but the converse does not hold. It is true, however, for mutually permutable products $G=A B$ such that $A \cap B=1$ [7, Proposition 3.5].

Asaad and Shaalan obtained in [3] results about totally and mutually permutable products of supersoluble groups. For totally permutable products, they obtained the following:

If $G$ is the totally permutable product of two supersoluble subgroups $A$ and $B$, then $G$ is supersoluble.

Moreover they proved that this result is not valid for mutually permutable products, but the following generalization [3, Theorem 3.1] of Baer's result is true:

If $G$ is the mutually permutable product of two supersoluble subgroups $A$ and $B$, and the derived subgroup $G^{\prime}$ of $G$ is nilpotent, then $G$ is supersoluble.

Finally, this last result remains valid if the nilpotency of $G^{\prime}$ is replaced by the nilpotency of one of the factors [3, Theorem 3.2].

In the last part of this paper some generalizations of these results will be naturally obtained from our study.

In recent years, some weaker versions of the concepts of totally and mutually permutable products have been introduced. Permutability of each factor with some specific families of subgroups of the other has often been analyzed. In this context, we study the structure of mutually $s n$-permutable products: 
Definition Let $A$ and $B$ be two subgroups of a group $G$ such that $G=A B$.

a) We say that $G=A B$ is the totally sn-permutable product of $A$ and $B$ if every subnormal subgroup of A permutes with every subnormal subgroup of $B$.

b) We say that $G=A B$ is the mutually sn-permutable product of $A$ and $B$ if $A$ permutes with every subnormal subgroup of $B$ and $B$ permutes with every subnormal subgroup of $A$.

Carocca showed [8, Theorem 6] that every group which can be factorized as a mutually sn-permutable product of two soluble groups is soluble as well. In the study of factorized groups in which each factor permutes with a specific family of subgroups of the other, the embedding of the intersection of the factors plays a very important role. In Section 2 we analyze this embedding and apply it to obtain an alternative proof of Carocca's result.

Since the purpose of this paper is to investigate the structure of a group $G=A B$ which is either a totally sn-permutable product or a mutually $s n$-permutable product of two supersoluble subgroups $A$ and $B$, we shall assume in the sequel that all groups are not only finite but also soluble, except of course in the statement and proof of Carocca's Theorem.

We obtain a supersoluble version of Stonehewer's Theorem by assuming that the product is mutually $s n$-permutable. In fact, we have the following stronger result:

Theorem A Let $G=A B$ be the mutually sn-permutable product of the supersoluble subgroups $A$ and $B$. If $N$ is a minimal normal subgroup of $G$, then both $A N$ and $B N$ are supersoluble.

This theorem turns out to be useful in the study of the supersolubility of $s n$-permutable products of supersoluble groups. It also allowed us to prove an important structural theorem for mutually permutable products of supersoluble groups (see [1]).

It is not true in general that a group which is the totally sn-permutable product of two supersoluble subgroups is supersoluble, as we shall see in the last section of this paper. However this result of Beidleman, Galoppo, Heineken and Manfredino ([6]) gives a sufficient condition for this property to be satisfied:

Theorem 1 Let $G=A B$ be a finite group which is the totally sn-permutable product of the subgroups $A$ and $B$. If $A$ is supersoluble and $B$ is nilpotent, then $G$ is supersoluble.

Note that the proof in [6] does not require the finiteness of $G$. On the other hand, this last Theorem confirms that the result of Asaad and Shaalan holds in the case of a totally $s n$-permutable product (instead of a totally 
permutable product) if we assume the nilpotency of one of the factors. We will see that a proof of this result in the finite case follows easily from Theorem A, as does the following Theorem:

Theorem B. Let $G=A B$ be the mutually sn-permutable product of the subgroups $A$ and $B$, where $A$ is supersoluble and $B$ is nilpotent. If $B$ permutes with each Sylow subgroup of $A$, then the group $G$ is supersoluble.

The hypothesis on $\mathrm{B}$ in the above theorem is essential in order to get supersolubility as we will show by means of an example in the last section of this paper.

Moreover, we prove that the second theorem of Asaad and Shaalan remains true if $G$ is a mutually $s n$-permutable product without any aditional requirement.

Theorem C. Let $G=A B$ be the mutually sn-permutable product of the supersoluble subgroups $A$ and $B$. If the derived subgroup $G^{\prime}$ of $G$ is nilpotent, then $G$ is supersoluble.

Notice that the group in Theorem $\mathrm{C}$ is nilpotent-by-abelian. Hence a natural question is whether or not a metanilpotent group which is the mutually sn-permutable product of two supersoluble subgroups is supersoluble. The second example in Section 5 answers that question negatively, but additional assumptions allow us to get supersolubility:

Theorem D. Let $G=A B$ be the mutually sn-permutable product of the supersoluble subgroups $A$ and $B$. Assume that $G$ is metanilpotent. If

$$
\left(\left|A / A^{\mathcal{N}}\right|,\left|B / B^{\mathcal{N}}\right|\right)=1
$$

then $G$ is supersoluble.

\section{Some properties of mutually sn-permutable products}

Lemma 1 Let $G=A B$ be a mutually sn-permutable product. Let $I=A \cap B$ and let $L$ be a subnormal subgroup of $A$ such that $I \leq L$. Then $L B$ is again a mutually sn-permutable product.

Proof. It is obvious that $B$ permutes with every subnormal subgroup of $L$. On the other hand, if we take $R$ a subnormal subgroup of $B$, then we have that $L R=L I R=L(A \cap B) R=(A \cap L B) R=A R \cap L B=R A \cap B L=$ $R(A \cap B L)=R(A \cap B) L=R L$. Therefore, we have that $L B$ is a mutually sn-permutable product.

Corollary 1 If $G=A B$ is a mutually sn-permutable product such that $A \cap B=1$, then $G=A B$ is a totally sn-permutable product.

Proof. This is a straightforward consequence of the previous lemma. 
Lemma 2 Let $G=A B$ be a mutually sn-permutable product of the subgroups $A$ and $B$. If $N$ is a minimal normal subgroup of $G$, then either $A \cap N=B \cap N=1$ or $N=(A \cap N)(B \cap N)$. In this second situation, the product $(A \cap N)(B \cap N)$ is mutually sn-permutable.

Proof. Clearly $A \cap N$ is a normal subgroup of $A$ and hence $X=(A \cap N) B$ is a subgroup of $G$ such that $N \cap X=N \cap(A \cap N) B=(A \cap N)(B \cap N)$. Now since $N \cap X$ is a normal subgroup of $X, B$ normalizes $N \cap X=(A \cap N)(B \cap N)$.

Arguing similarly, $Y=A(B \cap N)$ is a subgroup of $G$ such that $Y \cap N=$ $A(B \cap N) \cap N=(A \cap N)(B \cap N)$

As before, $Y$ (and hence $A$ ) normalizes $Y \cap N=(A \cap N)(B \cap N)$. We can conclude that $(A \cap N)(B \cap N)$ is a normal subgroup of $G$, and the minimality of $N$ implies that either $A \cap N=B \cap N=1$ or $N=(A \cap N)(B \cap N)$.

If this last situation holds, then given any subnormal subgroup $A_{0}$ of $A \cap N$ we have that $A_{0}$ is subnormal in $A$ as well and thus $A_{0} B \leq G$. But then $A_{0}(B \cap N)=A_{0} B \cap N \leq G$. A similar argument shows that $A \cap N$ permutes with every subnormal subgroup of $B \cap N$, and consequently the product $(A \cap N)(B \cap N)$ is mutually sn-permutable.

Following the notation used in [9, III.4 and IV.5], we define:

Definition. Let $G$ be a group, and let $\mathcal{X}$ be a class of groups. We say that a maximal subgroup $M$ is $\mathcal{X}$-normal in $G$ if $G / \operatorname{Core}_{G}(M) \in \mathcal{X}$.

If $\mathcal{F}$ is a saturated formation, a subgroup $H$ of $G$ is said to be $\mathcal{F}$ subnormal in $G$ if $H=G$ or there exists a chain of subgroups

$$
H=H_{0}<H_{1}<\cdots<H_{n}=G
$$

such that $H_{i-1}$ is a maximal $\mathcal{F}$-normal subgroup of $H_{i}$, for each $i=1,2, \ldots, n$.

$\mathcal{U}$-subnormality will appear in our context. An interesting connection which will link this embedding property with subnormal permutability can be stated in the following terms:

Theorem 2 Let $G$ be a group. If $H$ is a subgroup of $G$ such that $H$ permutes with every subnormal subgroup of $G$, then $H$ is $\mathcal{U}$-subnormal in $G$.

Proof. We assume that the theorem is false and let $G$ be a minimal counterexample. There exists a subgroup $H$ of $G$ which permutes with every subnormal subgroup of $G$ but is not $\mathcal{U}$-subnormal in $G$. Let $N$ be a minimal normal subgroup of $G$. Clearly $H N / N$ permutes with every subnormal subgroup of $G / N$. The minimality of $G$ yields that $H N / N$ is $\mathcal{U}$-subnormal in $G / N$ and therefore $H N$ is $\mathcal{U}$-subnormal in $G$ as a consequence of [10, Lemma 1.1]. 
Since $N$ is a minimal normal subgroup of the soluble group $G$, then $N$ must be an elementary abelian $p$-group, for some prime $p$. But $H$ permutes with every subnormal subgroup of $G$ and consequently also with every subgroup of $N$. In particular, there exists a series

$$
H<H N_{1}<\cdots<H N_{r}<H N
$$

such that $\left|H N: H N_{r}\right|=\left|H N_{j+1}: H N_{j}\right|=\left|H N_{1}: H\right|=p$, for each $j=1, \ldots, r-1$. This clearly implies that $H$ is a $\mathcal{U}$-subnormal subgroup of $H N$. Hence we have that $H$ is $\mathcal{U}$-subnormal in $G$, a contradiction.

As a consequence of the above Theorem and [5, Lemma 3.1], we have that if $H$ permutes with every subnormal subgroup of a group $G$, then the supersoluble residual of $H$ is subnormal in $G$.

Theorem 3 [8, Theorem 6] Let $G=A B$ be the mutually sn-permutable product of the subgroups $A$ and $B$ of $G$. If both $A$ and $B$ are soluble, then $G$ is also soluble.

Proof. Let us assume that the theorem is false and choose a minimal counterexample, $G$ say. Arguing by induction, we know that $G$ must have a unique minimal normal subgroup, which is non-abelian, $N$ say. Using Lemma 2, we know that either $N=(A \cap N)(B \cap N)$ or $A \cap N=B \cap N=1$. On the other hand, applying Theorem $2, A \cap B$ is an $\mathcal{U}$-subnormal subgroup of $A$ and $B$.

Take now a minimal normal subgroup $N_{1}$ of $A$ and a minimal normal subgroup $N_{2}$ of $B$, respectively. Then it is clear that $N \leq N_{1}^{G} \cap N_{2}^{G} \leq$ $N_{1} B \cap A N_{2}=\left[N_{1}(A \cap B)\right] N_{2}$. Moreover we can suppose that Core $_{A}(A \cap B)=$ Core $_{B}(A \cap B)=1$, otherwise we would take $N_{1}$ or $N_{2}$ contained in $A \cap B$ and $N$ would be contained in $A$ or $B$, a contradiction.

Let us assume firstly that $N=(A \cap N)(B \cap N)$. If $N \neq G$, then the solubility of $G / N$ follows from the minimality of $G$. But $N$ is again a mutually $s n$-permutable product of two soluble subgroups by Lemma 2, and therefore $N$ itself is soluble. It follows that $G$ is soluble, a contradiction. Consequently we can assume that $N=G$ and hence $G$ must be a simple group. Furthermore by the above arguments $N \leq N_{1}(A \cap B) N_{2}$. Consequently $G=N_{1}(A \cap B) N_{2}$ and hence $A=N_{1}(A \cap B)$. Notice that $N_{1}$ is a minimal normal subgroup of $A$. Hence $A \cap B$ is an $\mathcal{U}$-subnormal maximal subgroup of $A$ with $\operatorname{Core}_{A}(A \cap B)=1$. Therefore $A$ is supersoluble and $N_{1}$ is a cyclic group of prime order, $p$ say. Arguing in an analogous way we can assume also that $B$ is supersoluble and $N_{2}$ is a cyclic group of prime order, $q$ say. Since $\operatorname{Core}_{G}(A)=\operatorname{Core}_{G}(B)=1$, we have that $G$ is isomorphic to subgroups of the symmetric groups of degree $p$ and $q$ respectively. 
Consequently $p=q$. Moreover, $\left|N_{1} N_{2}\right|=p^{2}$, that is, $N_{1} N_{2}$ is an abelian subgroup of $G$. Recall that $G=N_{1}(A \cap B) N_{2}$. This means that $N_{1}$ is a normal subgroup of $G$, a contradiction.

Therefore we may assume for the rest of the proof that $A \cap N=B \cap N=1$. Take, as above, minimal normal subgroups $N_{1}$ of $A$ and $N_{2}$ of $B$. It is clear that $N_{1}$ and $N_{2}$ are a $p$-group and a $q$-group, respectively, for primes $p$ and $q$. Now if there exists a prime $r \neq p, r \neq q$ such that $r$ divides $|N|$, then every Sylow $r$-subgroup of $A \cap B$, say $H$, will be a Sylow $r$-subgroup of $N_{1}(A \cap B) N_{2}$ as well. But therefore $H \cap N$ is a Sylow $r$-subgroup of $N$. We reach a contradiction if we note that $H \cap N \leq A \cap B \cap N=1$. Hence $N$ is a $\{p, q\}$-group, and this implies the solubility of $N$ by Burnside's Theorem $[9$, Theorem I.2]. This fact leads to our final contradiction since $G / N$ is also soluble by induction.

\section{The "supersoluble version" of Stonehewer's theorem}

\section{Proof of Theorem A}

Assume the result is not true and let $G$ be a minimal counterexample. Let $N$ be a minimal normal subgroup of $G$ such that either $A N$ or $B N$ is not supersoluble. We know by Theorem 3 that $G$ must be soluble, and thus $N$ is an elementary abelian $p$-group for some prime $p$. We prove that $N$ is the unique normal minimal subgroup of $G$.

Let $M$ denote a minimal normal subgroup of $G$ with $N \neq M$. First the group $G / M$ satisfies the hypotheses of the theorem and hence the minimal choice of $G$ implies that both $A N M / M$ and $B N M / M$ are supersoluble. Therefore, the supersoluble residual $(A N)^{\mathcal{U}}$ of $A N$ is contained in $M$. Moreover, $A N / N$ is supersoluble. Hence $(A N)^{\mathcal{U}} \leq N$. Consequently $(A N)^{\mathcal{U}} \leq$ $M \cap N=1$, and $A N$ is supersoluble. Analogously, since $B N M / M$ is supersoluble, we can deduce that $B N$ is supersoluble, a contradiction. Consequently we may assume that $N=\operatorname{Soc}(G)$ is the unique minimal normal subgroup of $G$. Suppose that $N \leq A$. Then every subgroup of $N /(B \cap N)$ is normal in $B N /(B \cap N)$. This implies that $B N$ is supersoluble, a contradiction. Consequently $N$ is neither contained in $A$ nor in $B$.

We shall see next that $p$ must be the largest prime dividing $|G|$. Let $q$ be this largest prime, and assume $q \neq p$. We can assume that $q$ divides $|B|$. In such case, $B$ has a unique Sylow $q$-subgroup, $B_{q}$ say. Note that $A B_{q}$ is a subgroup of $G$. Now $1 \neq B_{q}^{G}=B_{q}^{B A}=B_{q}^{A} \leq A B_{q}$ and thus $N$ is contained in $B_{q}^{G}$. This clearly implies that $N \leq A$, a contradiction.

Using Lemma 2, we know that either $N=(A \cap N)(B \cap N)$ or $A \cap N=$ $B \cap N=1$. We consider these two possible situations separately. 
Let us assume first that $N=(A \cap N)(B \cap N)$. Note that we can assume that both $A \cap N$ and $B \cap N$ are non-trivial proper subgroups of $N$.

We know that either $A N$ or $B N$ is not supersoluble. We assume without loss of generality that $Z=A N$ is not supersoluble. Let $A_{1}$ be a supersoluble projector of $Z$ containing $A$ (see [9; III, 3]). Note that $Z=A_{1} Z^{\mathcal{U}}$ and $1=\left(A_{1} \cap Z^{\mathcal{U}}\right) \neq Z^{\mathcal{U}} \leq N$ (see [9; IV, 5.18]). Let $x$ be a non-trivial element of $Z^{\mathcal{U}}$. We can find elements $n_{1}$ of $N \cap A$ and $n_{2}$ of $N \cap B$ such that $x=n_{1} n_{2}$ and $o\left(n_{i}\right)=p$ for $i=1,2$. If $\left\langle n_{2}\right\rangle \cap A_{1} \neq 1$ then $n_{2} \in A_{1}$ and thus $x \in Z^{\mathcal{U}} \cap A_{1}=1$, a contradiction. Consequently $\left\langle n_{2}\right\rangle \cap A_{1}=1$. Moreover, $A_{1}=A\left(N \cap A_{1}\right)$. Since $\left\langle n_{2}\right\rangle$ permutes with both $A$ and $N \cap A_{1}$, it follows that $\left\langle n_{2}\right\rangle$ permutes with $A_{1}$. Therefore $X=A_{1}\left\langle n_{2}\right\rangle$ is a subgroup of $G$. Note that

$$
N \cap X=N \cap A_{1}\left\langle n_{2}\right\rangle=\left\langle n_{2}\right\rangle\left(N \cap A_{1}\right)
$$

is a normal subgroup of $X$ as is $N \cap A_{1}$. If we consider the series $N \cap$ $A_{1} \unlhd\left\langle n_{2}\right\rangle\left(N \cap A_{1}\right) \unlhd X$, we can deduce that $X / N \cap A_{1}$ is a supersoluble group. Hence $X^{\mathcal{U}}$ is contained in $A_{1} \cap Z^{\mathcal{U}}=1$ and consequently $X$ is supersoluble. This is impossible since $A_{1}$ is a supersoluble projector of $X$.

Hence we can assume that $A \cap N=B \cap N=1$. We now prove:

Given any non-trivial element $x$ of $N$, there must exist a subgroup $N_{x}$ of $N$ such that $x \in N_{x}, N_{x} \unlhd A N_{x}$ and $A N_{x}$ is supersoluble.

In order to prove this statement, note that if $A_{p}$ and $B_{p}$ denote the Sylow $p$-subgroups of $A$ and $B$ respectively, then $N \leq A_{p} B_{p}$ applying [2; Corollary 1.3.3]. Consequently we can write $x=a b$ where $a \in A_{p}$ and $b \in B_{p}$. Note that $\langle b\rangle$ is a subnormal subgroup of $B$ and therefore it permutes with $A$. Let $Y=A\langle b\rangle$, and write $N_{x}=N \cap Y$. Clearly $x \in N_{x} \leq N$ and $N_{x}$ is a normal subgroup of $Y=A(N \cap Y)=A N_{x}$. We shall be done if $Y$ is supersoluble. Assume on the contrary that $Y$ is not supersoluble and take a supersoluble projector $A_{1}$ of $Y$ with $A \leq A_{1}$ (see [9; III, 3]). Let now $\beta$ be the smallest non-negative integer such that $b^{p^{\beta}} \in A_{1}$. Since $Y=A\langle b\rangle=A_{1}\langle b\rangle$ and $Y$ is not supersoluble, we have that $\beta>0$. Moreover, $A_{1} \cap\langle b\rangle=\left\langle b^{p^{\beta}}\right\rangle$. Write $T=A_{1}\left\langle b^{p^{\beta-1}}\right\rangle=A_{1} T^{\mathcal{U}}$. Note that $A_{1}$ is a supersoluble projector of $T$, and that $T^{\mathcal{U}}$ is an abelian group since $T^{\mathcal{U}} \leq Y^{\mathcal{U}}$, and $T^{\mathcal{U}} \cap A_{1}=1$. Now

$$
\left|T^{\mathcal{U}}\right|=\left|T: A_{1}\right|=\left|\left\langle b^{p^{\beta-1}}\right\rangle:\left\langle b^{p^{\beta}}\right\rangle\right|=p
$$

and therefore $T$ is supersoluble, a contradiction. Hence we have proved the above statement.

Assume now that $A N$ is not supersoluble. There must exist, in such case, a non-cyclic chief factor $K / L$ of $A N$, with $K \leq N$. Let $x \in K \backslash L$. Following 
the above claim, we know that there must exist a subgroup $N_{x}$ of $N$ in the described situation. Note that $N_{x}$ is in fact a normal subgroup of $A N$. Now $N_{x} L / L$ is a normal subgroup of $A N / L$ and $K / L$ is a minimal normal of $A N / L$. Consequently $1 \neq x L \in N_{x} L / L \cap K / L$. Thus $N_{x} L / L \cap K / L \neq 1$ and hence $K / L \leq N_{x} L / L \leq A N_{x} / L$ which is supersoluble. We conclude that $K / L$ is a cyclic group, our final contradiction.

\section{Main consequences}

The following lemma will be used in the proofs of this section.

Lemma 3 Let $G$ be a primitive group and let $N$ be its unique minimal normal subgroup. Assume that $G / N$ is supersoluble. If $N$ is a p-group, where $p$ is the largest prime dividing $|G|$, then $N=F(G)=O_{p}(G)$ is a Sylow p-subgroup of $G$.

Proof. It is well known that $N=F(G)=O_{p}(G)$ since $O_{q}(G)=1$ for every prime $q \neq p$. Therefore we only need to show that $N$ is a Sylow $p$-subgroup of $G$.

Since $G$ is a primitive soluble group, we can write $G=N M$, where $M$ is a maximal subgroup of $G, N \cap M=1$ and $C_{G}(N)=N$.

Note that $O_{p}(M)=1$ by [9, Theorem A.15.6.b]. Clearly $M \cong G / N$ is supersoluble. On the other hand, a Sylow $p$-subgroup of $M$ is normal in $M$ and consequently $p$ does not divide the order of $M$. This completes the proof.

We begin by studying a minimal counterexample to claim the following:

"If $G=A B$ is the mutually sn-permutable product of the supersoluble subgroups $A$ and $B$, then $G$ is supersoluble."

Let $G=A B$ be a minimal counterexample to the above property. $G$ must be a primitive soluble group, and hence there exists a unique minimal normal subgroup $N$ of $G$, such that $N=C_{G}(N)$. Let $p$ be the prime dividing $|N|$. By using Theorem A, we know that both $A N$ and $B N$ are supersoluble.

We show that $p$ must be the largest prime dividing $|G|$. Let $q$ be this largest prime, and assume $q \neq p$. We can assume without loss of generality that $q$ divides $|A N|$. The supersoluble group $A N$ must have a unique Sylow $q$-subgroup, $(A N)_{q}$ say, and clearly $(A N)_{q}$ centralizes $N$. This implies that $(A N)_{q}=1$ since $C_{G}(N)=N$, a contradiction. Thus $p$ must be the largest prime dividing $|G|$ and so we have the structure given by Lemma 3 .

Next we consider a minimal counterexample to the following claim:

"Let $G=A B$ be the mutually sn-permutable product of the subgroups $A$ and $B$, where $A$ is supersoluble and $B$ is nilpotent. Then $G$ is supersoluble." 
Consider such a minimal counterexample, $G$ say. As before, $G=A B$ is a primitive soluble group with a unique minimal normal subgroup $N . N$ is a self-centralizing $p$-group for a prime $p$. By the argument above and by Lemma 3,p must be the largest prime dividing $G, N$ is the unique Sylow $p$-subgroup of $G$ and by Theorem A both $A N$ and $B N$ are supersoluble.

We shall see now that $N$ must be contained in $A$. Note that if $N \leq B$, then the nilpotency of $B$ implies that $B$ is a $p$-group. In such case $G=A N$ and $G$ is supersoluble, a contradiction. Consequently we can assume that $N$ is not contained in $B$. Consider now a Hall $p^{\prime}$-subgroup $B_{p^{\prime}}$ of $B$. Since $B_{p^{\prime}}$ is a normal subgroup of $B$, it permutes with $A$ and thus $A B_{p^{\prime}}$ is a subgroup of $G$. If $B_{p^{\prime}} \neq 1$ then $1 \neq B_{p^{\prime}}^{G} \leq A B_{p^{\prime}}$ and hence $N \leq A$, as desired. Hence $B_{p^{\prime}}=1$ and thus $B$ must be a $p$-group. In such case $B \leq N$ and hence $G=A B=A N$ and $G$ is supersoluble, a contradiction. Thus $A \cap N=N$ and therefore $N \leq A$, as we wanted to prove.

At this point we easily obtain the following consequences:

Proof of Theorem 1 [6, Theorem 2]

From the argument above, we can assume that $N \leq A$, where $N$ denotes the unique minimal normal subgroup of $G, N$ an elementary abelian Sylow $p$-subgroup of $G$. Denote by $N_{1}$ a minimal normal subgroup of $A$ such that $N_{1} \leq N$. Then $N_{1}$ permutes with $B_{p^{\prime}}$ and is centralized by $B_{p}$. Hence $N \leq N_{1}^{G} \leq N_{1} B_{p^{\prime}}$ and so $N \leq N_{1}$. Consequently $N=N_{1}$ and $N$ is a cyclic group of prime order, the final contradiction.

\section{Proof of Theorem B}

As $B$ permutes with every Sylow subgroup of $A$, we can consider the subgroup of $G, A_{p^{\prime}} B$, where $A_{p^{\prime}}$ denotes a Hall $p^{\prime}$-subgroup of $A$. Notice that if $A_{p^{\prime}}=1$, we would have $A=N$, and $G=B N$ is supersoluble by Theorem A, a contradiction. If $G=A_{p^{\prime}} B$, then $N \leq B$ and $B$ is a $p$-group, a contradiction. Hence $A_{p^{\prime}} B<G$ and $G=N A_{p^{\prime}} B$. On the other hand, $N \cap A_{p^{\prime}} B=N \cap B$ is a normal subgroup of $A_{p^{\prime}} B$. Hence $N \cap B$ is a normal subgroup of $G, N=N \cap B \leq B$ and consequently $N \cap B=1$. Therefore $B$ is a $p^{\prime}$-group. Take now $N_{1}$ a minimal normal subgroup of $A$ with $N_{1} \leq N$. Then $N \leq N_{1}^{G} \leq N_{1} B \leq G$. Thus $N=N_{1}$, a cyclic group of prime order, the final contradiction.

\section{Proof of Theorem C}

Assume the result is not true and let $G$ be a minimal counterexample. It is clear that $G^{\prime} \neq 1$ and that $G$ is a primitive group. Let $N$ be the unique minimal normal subgroup. By Lemma 3, we have $N=F(G)$ and so $G^{\prime} \leq F(G)=N$. From $A N$ and $B N$ are supersoluble (by Theorem A) and normal $\left(G^{\prime} \leq A N, G^{\prime} \leq B N\right)$ we can apply Baer's Theorem to give $G$ supersoluble, a contradiction. 


\section{Proof of Theorem D}

Assume that the Theorem is false and take a minimal counterexample $G=A B$. We have that $G$ is a primitive soluble group. Write, as usual, $G=N M$, where $N$ is the unique minimal normal subgroup of $G, M$ is a maximal subgroup of $G, N \cap M=1$ and $C_{G}(N)=N$. We also know that $N$ is a $p$-group for some prime $p$, and by Lemma 3 and the metanilpotence of $G$ we have $N=F(G)=O_{p}(G)=G^{\mathcal{N}}$ and $N$ is a Sylow $p$-subgroup of $G$. Consequently we may assume that $M \cong G / N$ is nilpotent. Note that $O_{p}(M)=1$ by $[9$, Theorem A.15.6.b].

Let now $A_{p^{\prime}}$ and $B_{p^{\prime}}$ be Hall $p^{\prime}$-subgroups of $A$ and $B$, respectively, such that $A_{p^{\prime}} B_{p^{\prime}}$ is a Hall $p^{\prime}$-subgroup of $G$ (see [2, Theorem 1.3.2]). Replacing $M$ by some conjugate if necessary, we can assume $A_{p^{\prime}} B_{p^{\prime}}=M$. The nilpotency of $M$ and the fact that $\pi(|A|) \cap \pi(|B|) \subseteq\{p\}$ allows us to write $M=A_{p^{\prime}} \times B_{p^{\prime}}$.

Take now the subgroup $T=B N$ of $G$. Note that $T$ is supersoluble according to Theorem A. Using the local definition of the class of supersoluble groups, we obtain that $T / O_{p^{\prime} p}(T)$ is an abelian group of exponent dividing $p-1$.

Now $N$ is self-centralizing in $T$ and hence $O_{p^{\prime}}(T)=1$. It follows that $O_{p^{\prime} p}(T)=O_{p}(T)=O_{p}(B N)=N$. This implies that $B N / N \cong B / B \cap N \cong$ $B_{p^{\prime}}$ is an abelian group of exponent dividing $p-1$. We could reproduce the same argument for $A$ and obtain thus that both $A_{p^{\prime}}$ and $B_{p^{\prime}}$ are abelian groups of exponent dividing $p-1$.

But $M=A_{p^{\prime}} \times B_{p^{\prime}}$, and hence $M$ is also abelian of exponent dividing $p-1$. But $N$ is an irreducible and faithful module for $M$, and consequently its dimension must equal one. We conclude that $N$ is cyclic, the final contradiction.

\section{Some counterexamples and remarks}

It is not true in general that a group which is the totally sn-permutable product of two supersoluble subgroups is supersoluble, as the following example shows.

Example. Let $V$ be a faithful irreducible module for $S$, the symmetric group of degree 3, over the field of 7 elements. It is easy to check that $V$ has dimension 2. Let $G$ be the semidirect product of $V$ and $S$, and let $A=V S_{3}$, $B=V S_{2}$, where $S_{p}$ is a Sylow $p$-subgroup of $S$. It is again easy to check that $A$ and $B$ are supersoluble, $G$ is the totally sn-permutable product of $A$ and $B$, but $G$ is not supersoluble. 
Note that the hypothesis on $B$ in Theorem $\mathrm{B}$ is essential in order to get the supersolubility of $G$ as the following example illustrates.

Example. Consider $X=\left\langle a, b: a^{4}=1=b^{2}, a^{b}=a^{-1}\right\rangle$, the dihedral group of order 8, and let $V=\left\langle v_{1}, v_{2}\right\rangle$ be a $G F(5)$-space of dimension 2. Then $V$ can be considered as an $X$-module with the following action:

$$
\begin{array}{ll}
v_{1}^{a}=3 v_{1} & v_{1}^{b}=v_{2} \\
v_{2}^{a}=2 v_{2} & v_{2}^{b}=v_{1}
\end{array}
$$

Take now $G=[V] X$, the corresponding semidirect product, and consider the following subgroups of $G$ :

$$
A=V\langle a\rangle ; B=\left\langle v_{1} v_{2}\right\rangle \times\langle b\rangle
$$

Note that $G=A B$. It is clear that $A$ is supersoluble, $B$ is nilpotent and the nilpotent residual of $G$ is precisely $V$, which is abelian. It is easy to see that $G$ is the mutually $s n$-permutable product of the subgroups $A$ and $B$, but $G$ is not supersoluble.

\section{References}

[1] Alejandre, Manuel J., Ballester-Bolinches, A., Cossey, John: Permutable products of supersoluble groups. To appear in J. Algebra.

[2] Amberg, B., Franciosi, S. and De Giovanni, F.: Products of groups. Oxford Mathematical Monographs. The Clarendon Press, Oxford University Press, New York, 1992.

[3] Asand, M., Shanlan, A.: On the supersolubility of finite groups. Arch. Math. 53 (1989), no. 4, 318-326.

[4] BAER, R.: Classes of finite groups and their properties. Illinois J. Math. 1 (1957), 115-187.

[5] Ballester-Bolinches, A., Doerk, K. and Pérez-Ramos, M. D.: On the lattice of $\mathcal{F}$-subnormal groups. J. Algebra 148 (1992), no. 1, 42-52.

[6] Beidleman, J. C., Galoppo, A., Heineken, H. and Manfredino, M.: On certain products of soluble groups. Forum Math. 13 (2001), 569-580.

[7] Carocca, A.: $p$-supersolvability of factorized finite groups. Hokkaido Math. J. 21 (1992), 395-403.

[8] Carocca, A.: On factorized finite groups in which certain subgroups of the factors permute. Arch. Math. 71 (1998), 257-261.

[9] Doerk, K. and Hawkes, T. O.: Finite soluble groups. De Gruyter Expositions in Mathematics 4. Walter de Gruyter, Berlin, 1992.

[10] Förster, P.: Finite groups all whose subgroups are $\mathcal{F}$-subnormal or $\mathcal{F}$ subabnormal. J. Algebra 103 (1986), no. 1, 285-293. 
[11] Kegel, O. H.: Sylow-Gruppen und Subnormalteiler endlicher Gruppen. Math. Z. 78 (1962), 205-221.

[12] Stonehewer, S. E.: On finite dinilpotent groups. J. Pure Appl. Algebra 88 (1993), no. 1-3, 239-244.

Recibido: 13 de mayo de 2002

Manuel J. Alejandre

Centro de Investigación Operativa

Universidad Miguel Hernández

Avda. del Ferrocarril s/n 03202 Elche (Spain)

m.alejandre@umh.es

A. Ballester-Bolinches

Departament d'Àlgebra

Universitat de València

Dr. Moliner 50

46100 Burjassot, València (Spain)

Adolfo.Ballester@uv.es

John Cossey

Mathematics Department

School of Mathematical Sciences

Australian National University

0200 Canberra (Australia)

John. Cossey@maths . anu . edu . au

M. C. Pedraza-Aguilera

Escuela Universitaria de Informática

Departamento de Matemática Aplicada

Universidad Politécnica de Valencia

Camino de Vera, s/n 46071 Valencia (Spain)

mpedraza@mat.upv.es

This paper was partly done while the first author was a visitor at the Australian National University. He is grateful to the Department of Mathematics for its warm hospitality while this investigation was carried out. The second and the fourth authors are supported by Proyecto PB 97-0674-C02-02 of DGICYT, MEC, Spain. The third author wishes to thank the Centro de Investigación Operativa of the Universidad Miguel Hernández for its support of this research. 